\title{
Nonlinear Tracking and Landing Controller for Quadrotor Aerial Robots
}

\author{
Holger Voos, Haitham Bou-Ammar \\ University of Applied Sciences Ravensburg-Weingarten, Mobile Robotics Lab, \\ 88241 Weingarten, Germany, Email: voos@ hs-weingarten.de
}

\begin{abstract}
Quadrotor UAVs are one of the most preferred type of small unmanned aerial vehicles because of the very simple mechanical construction and propulsion principle. However, the nonlinear dynamic behavior requires a more advanced stabilizing control and guidance of these vehicles. In addition, the small payload reduces the amount of batteries that can be carried and thus also limits the operating range of the UAV. One possible solution for a range extension is the application of a base station for recharging purpose even during operation. In order to increase the efficiency of the overall system further, a mobile base station will be applied here. However, landing on a moving base station requires autonomous tracking and landing control of the UAV. In this paper, a novel nonlinear autopilot for quadrotor UAVs is extended with a tracking and landing controller to fulfil the required task. First simulation and experimental results underline the performance of this new control approach for the current realization.
\end{abstract}

\section{INTRODUCTION}

Unmanned aerial vehicles (UAVs) already have a wide area of possible applications. Recent results in miniaturization, mechatronics and microelectronics also offer an enormous potential for small and inexpensive UAVs for commercial use. While many possible types of small UAVs exist, one very promising vehicle with respect to size, weight and maneuverability is the so called quadrotor. The quadrotor is a system with four propellers in a cross configuration, see Fig. 1 for a sketch of a quadrotor UAV. While the front and the rear motor rotate clockwise, the left and the right motor rotate counter-clockwise which nearly cancels gyroscopic effects and aerodynamic torques in trimmed flight. One additional advantage of the quadrotor compared to a conventional helicopter is the simplified rotor mechanics. By varying the speed of the single motors, the lift force can be changed and vertical and/or lateral motion can be generated. However, in spite of the four actuators, the quadrotor is a dynamically unstable nonlinear system that has to be stabilized by a suitable control system.

One main drawback of small UAVs in nearly all types of application is the reduced payload which also limits the amount of batteries that can be carried. Therefore the UAV has to return to a base station after a comparatively short amount of time for recharging purpose. In addition, in order to fulfill missions where a longer operating range is required such as pipeline, border or coast surveillance, returning to a stationary base station is also not useful. In such applications it would be more suitable to operate with an autonomous mobile base station that is able to carry a higher amount of energy for several recharging cycles. Such a mobile base station could be an autonomous mobile robot or ship. Coordinated parallel operation of the mobile base station and the UAV then leads to an overall system for aerial surveillance with extended range. However, that concept requires basic stabilizing control of the quadrotor, tracking of the mobile base station and finally control of the landing procedure.

In this paper, we first address the problem of a precise and fast stabilization of the quadrotor UAV since the fulfillment of this task is a precondition for further implementation of other functionalities. In spite of the four actuators, the quadrotor is a dynamically unstable system with nonlinear dynamics that has to be stabilized by a suitable control system. Concerning controller design for small quadrotor UAVs, some solutions are already proposed in the literature, see e.g. [1], [2], [3], [4] and [5] to mention only a few. Many of the proposed control systems are based on a linearized model and conventional PID- or state space control while other approaches apply sliding-mode, $\mathrm{H}_{\infty}$ or SDRE control [4], [5]. Recently, a new nonlinear control algorithm has been proposed by the author which is based upon a decomposition of the overall controller into a nested structure of velocity and attitude control, see [6]. The controller has the advantage of an easy implementation and proven stability while taking the nonlinearities of the dynamics directly into account. Here, this controller is first shortly explained in order to provide the basis for the development of the nonlinear tracking and landing controller. This control strategies are then derived in details, first simulation and experimental results underline the obtained performance.

\section{DYNAMIC MODEL OF THE QUADROTOR}

The general dynamic model of a quadrotor UAV has been presented in a number of papers, see e.g. [1], [3], [4], [5] or [6], and therefore will not be discussed here in all details again. We consider an inertial frame and a body fixed frame whose origin is in the center of mass of the quadrotor, see Fig. 1. The attitude of the quadrotor is given by the roll, pitch and yaw angle, forming the vector $\boldsymbol{\Omega}^{T}=(\phi, \theta, \psi)$, while the position of the vehicle in the inertial frame is given by the position vector $\boldsymbol{r}^{T}=(x, y, z)$. The dynamic model of the quadrotor can be derived by applying the laws of conservation of momentum and angular momentum, taking the applied forces and torques into account (see [6]). The thrust force generated by rotor $i, i=1,2,3,4$ is $F_{i}=b \cdot \omega_{i}^{2}$ whith the thrust factor $b$ and the rotor speed $\omega_{i}$, and the law 


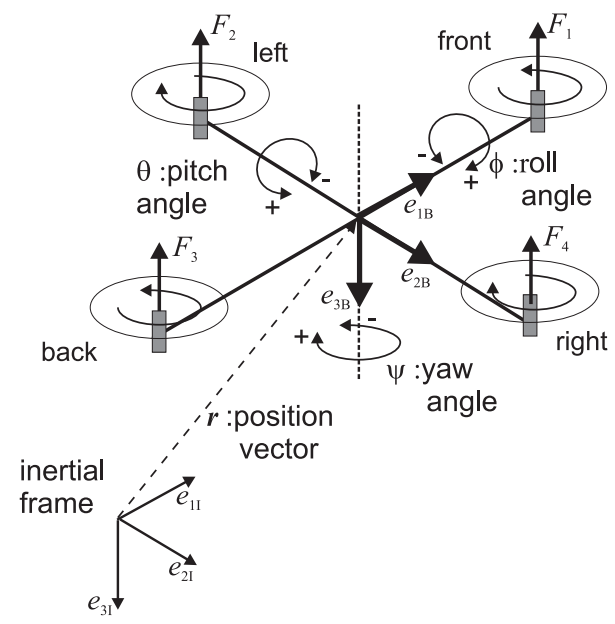

Fig. 1. Configuration, inertial and body fixed frame of the quadrotor.

of conservation of momentum yields

$$
\ddot{\boldsymbol{r}}=g \cdot\left(\begin{array}{l}
0 \\
0 \\
1
\end{array}\right)-\boldsymbol{R}(\boldsymbol{\Omega}) \cdot b / m \sum_{i=1}^{4} \omega_{i}^{2} \cdot\left(\begin{array}{l}
0 \\
0 \\
1
\end{array}\right)
$$

Herein, $\boldsymbol{R}(\boldsymbol{\Omega})$ is a suitable rotation matrix. With the inertia matrix $I$ (a pure diagonal matrix with the inertias $I_{x}, I_{y}$ and $I_{z}$ on the main diagonal), the rotor inertia $J_{R}$, the vector $M$ of the torque applied to the vehicle's body and the vector $M_{G}$ of the gyroscopic torques of the rotors, the law of conservation of angular momentum yields:

$$
I \ddot{\mathbf{\Omega}}=-(\dot{\boldsymbol{\Omega}} \times I \dot{\boldsymbol{\Omega}})-M_{G}+M
$$

The vector $M$ is defined as (see Fig. 1)

$$
\boldsymbol{M}=\left(\begin{array}{c}
L b\left(\omega_{2}^{2}-\omega_{4}^{2}\right) \\
L b\left(\omega_{1}^{2}-\omega_{3}^{2}\right) \\
d\left(\omega_{1}^{2}+\omega_{3}^{2}-\omega_{2}^{2}-\omega_{4}^{2}\right)
\end{array}\right)
$$

with the drag factor $d$ and the length $L$ of the lever. The gyroscopic torques caused by rotations of the vehicle with rotating rotors are

$$
\boldsymbol{M}_{G}=I_{R}\left(\dot{\boldsymbol{\Omega}} \times\left(\begin{array}{l}
0 \\
0 \\
1
\end{array}\right)\right) \cdot\left(\omega_{1}-\omega_{2}+\omega_{3}-\omega_{4}\right)
$$

The four rotational velocities $\omega_{i}$ of the rotors are the real input variables of the vehicle, but for a simplification of the model, the following substitute input variables are defined:

$$
\begin{aligned}
& u_{1}=b\left(\omega_{1}^{2}+\omega_{2}^{2}+\omega_{3}^{2}+\omega_{4}^{2}\right) \\
& u_{2}=b\left(\omega_{2}^{2}-\omega_{4}^{2}\right) \\
& u_{3}=b\left(\omega_{1}^{2}-\omega_{3}^{2}\right) \\
& u_{4}=d\left(\omega_{1}^{2}+\omega_{3}^{2}-\omega_{2}^{2}-\omega_{4}^{2}\right)
\end{aligned}
$$

Defining $\boldsymbol{u}^{T}=\left(u_{1}, u_{2}, u_{3}, u_{4}\right)$ and $\left(\omega_{1}-\omega_{2}+\omega_{3}-\omega_{4}\right)=$ $g(\boldsymbol{u})$ and introducing the vector of state variables $\boldsymbol{x}^{T}=$ ( $\dot{x}, \dot{y}, \dot{z}, \phi, \theta, \psi, \dot{\phi}, \dot{\theta}, \dot{\psi})$, evaluation of (1) until (5) yields the following state variable model:

$$
\dot{\boldsymbol{x}}=\left(\begin{array}{l}
-\left(\cos x_{4} \sin x_{5} \cos x_{6}+\sin x_{4} \sin x_{6}\right) \cdot u_{1} / m \\
-\left(\cos x_{4} \sin x_{5} \sin x_{6}-\sin x_{4} \cos x_{6}\right) \cdot u_{1} / m \\
g-\left(\cos x_{4} \cos x_{5}\right) \cdot u_{1} / m \\
x_{7} \\
x_{8} \\
x_{9} \\
x_{8} x_{9} I_{1}-\frac{I_{R}}{I_{x}} x_{8} g(\boldsymbol{u})+\frac{L}{I_{x}} u_{2} \\
x_{7} x_{9} I_{2}+\frac{I_{R}}{I_{y}} x_{7} g(\boldsymbol{u})+\frac{L}{I_{y}} u_{3} \\
x_{7} x_{8} I_{3}+\frac{1}{I_{z}} u_{4}
\end{array}\right)
$$

Herein, we use the abbreviations $I_{1}=\left(I_{y}-I_{z}\right) / I_{x}, I_{2}=$ $\left(I_{z}-I_{x}\right) / I_{y}$ and $I_{3}=\left(I_{x}-I_{y}\right) / I_{z}$. It becomes obvious that the state variable model can be decomposed into one subset of differential equations (DEQs) that describe the dynamics of the attitude using the last six equations of (6), and one subset that describes the translation of the UAV using the first three equations of (6).

\section{VEHICLE CONTROLLER DESIGN}

The task of the vehicle controller is the stabilization of a desired velocity vector which is calculated by the higherlevel mission controller. The decomposed structure of the state variable model (6) already suggests a nested structure for vehicle control. In order to achieve and maintain a desired velocity vector, first the necessary attitude of the UAV has to be stabilized. Therefore, we propose a decomposition of the vehicle control system in an outer-loop velocity control and an inner-loop attitude control system. In this structure, the inner attitude control loop has to be much faster than the outer loop and stabilizes the desired angles $\boldsymbol{\Omega}_{d}^{T}=$ $\left(\phi_{d}, \theta_{d}, \psi_{d}\right)=\left(x_{4, d}, x_{5, d}, x_{6, d}\right)$ that are commanded by the outer loop. First we consider the inner attitude control loop, then we derive the outer-loop controller to stabilize a desired velocity vector.

\section{A. Attitude Control System}

For the design of the attitude control system we consider the last six DEQs of (6) as the relevant submodel. Herein, the last three DEQs describing $x_{7}, x_{8}, x_{9}$ are nonlinear and depend on the input variables $u_{2}, u_{3}, u_{4}$, while $x_{4}, x_{5}, x_{6}$ are obtained from the former state variables by pure integration leading to three simple linear DEQs in (6). The control strategy now is as follows: we first apply a nonlinear feedback linearization to the last three DEQs in order to transfer them into linear and decoupled DEQs. Together with the set of the remaining linear DEQs we finally obtain three independent linear systems which can be stabilized via linear feedback.

If we first neglect the gyroscopic terms (since the rotor inertias are comparatively small) we obtain the simplified DEQs for $x_{7}, x_{8}, x_{9}$ as

$$
\left(\begin{array}{c}
\dot{x}_{7} \\
\dot{x}_{8} \\
\dot{x}_{9}
\end{array}\right)=\left(\begin{array}{c}
x_{8} x_{9} I_{1}+\frac{L}{I_{x}} u_{2} \\
x_{7} x_{9} I_{2}+\frac{L}{I_{y}} u_{3} \\
x_{7} x_{8} I_{3}+\frac{1}{I_{z}} u_{4}
\end{array}\right)
$$


Now we apply a feedback linearization in order to obtain a linear system:

$$
\begin{aligned}
& u_{2}=f_{2}\left(x_{7}, x_{8}, x_{9}\right)+u_{2}^{*} \\
& u_{3}=f_{3}\left(x_{7}, x_{8}, x_{9}\right)+u_{3}^{*} \\
& u_{4}=f_{4}\left(x_{7}, x_{8}, x_{9}\right)+u_{4}^{*}
\end{aligned}
$$

with the new input variables $u_{2}^{*}, u_{3}^{*}, u_{4}^{*}$. It can be shown that

$$
\begin{aligned}
f_{2}\left(x_{7}, x_{8}, x_{9}\right) & =\frac{I_{x}}{L}\left(K_{2} x_{7}-x_{8} x_{9} I_{1}\right) \\
f_{3}\left(x_{7}, x_{8}, x_{9}\right) & =\frac{I_{y}}{L}\left(K_{3} x_{8}-x_{7} x_{9} I_{2}\right) \\
f_{4}\left(x_{7}, x_{8}, x_{9}\right) & =I_{z}\left(K_{4} x_{9}-x_{7} x_{8} I_{3}\right)
\end{aligned}
$$

with the so far undetermined constant parameters $K_{2}, K_{3}, K_{4}$ transfer (7) into a set of linear and decoupled DEQs. It has been proven in [6] using a suitable Lyapunov function that this feedback is stable for $K_{2}, K_{3}, K_{4}<0$ even if the gyroscopic terms from (6) are considered again. Since $\dot{x}_{4}=x_{7}, \dot{x}_{5}=x_{8}, \dot{x}_{6}=x_{9}$ we finally obtain linear decoupled DEQs for $x_{4}, x_{5}, x_{6}$, respectively, see e.g. $x_{4}$ :

$$
\ddot{x}_{4}=K_{2} \dot{x}_{4}+L / I_{x} u_{2}^{*}
$$

If $x_{4 d}$ is the desired angle, application of a linear controller $u_{2}^{*}=w_{2} \cdot\left(x_{4 d}-x_{4}\right)$ with constant parameter $w_{2}$ leads to a closed-loop system of second order

$$
F(s)=\frac{X_{4}(s)}{X_{4 d}(s)}=\frac{w_{2}}{I_{x} / L \cdot s^{2}-K_{2} I_{x} / L \cdot s+w_{2}}
$$

The same considerations hold for the other angles with linear controllers $u_{3}^{*}=w_{3} \cdot\left(x_{5 d}-x_{5}\right)$ and $u_{4}^{*}=w_{4} \cdot\left(x_{6 d}-\right.$ $\left.x_{6}\right)$, respectively. The dynamics of these closed-loop systems now can be easily adjusted by a choice of a suitable set of parameters $\left(K_{2}, w_{2}\right),\left(K_{3}, w_{3}\right),\left(K_{4}, w_{4}\right)$, respectively, with the only limitation that $K_{2}, K_{3}, K_{4}<0$, see [6].

\section{B. Velocity Control System}

We now assume that the previously defined inner attitude control loops are adjusted in a way that their dynamic behavior is very fast compared to the outer velocity control loops. Therefore we approximate the inner closed control loops as static blocks with transfer function $F_{i}(s)=X_{i}(s) / X_{i d}(s) \approx$ $1, i=4,5,6$. Inserting this in (6), the velocities of the quadrotor UAV then can be approximated by

$\dot{x}_{1}=-\left(\cos x_{4 d} \sin x_{5 d} \cos x_{6 d}+\sin x_{4 d} \sin x_{6 d}\right) \cdot u_{1} / m$

$\dot{x}_{2}=-\left(\cos x_{4 d} \sin x_{5 d} \sin x_{6 d}-\sin x_{4 d} \cos x_{6 d}\right) \cdot u_{1} / m$

$\dot{x}_{3}=g-\cos x_{4 d} \cos x_{5 d} \cdot u_{1} / m$

where all $x_{4 d}, x_{5 d}, x_{6 d}$ and $u_{1}$ can be considered as input variables. Equation (12) can be interpreted in a way that all differential equations are of the form

$$
\left(\begin{array}{c}
\dot{x}_{1} \\
\dot{x}_{2} \\
\dot{x}_{3}
\end{array}\right)=\boldsymbol{f}\left(x_{4 d}, x_{5 d}, x_{6 d}, u_{1}\right)=\left(\begin{array}{c}
\tilde{u}_{1} \\
\tilde{u}_{2} \\
\tilde{u}_{3}
\end{array}\right)
$$

with the new input variables $\tilde{u}_{1}, \tilde{u}_{2}, \tilde{u}_{3}$ that depend on the other four input variables in a nonlinear form described by the vector function $f$. However, regarding these new input variables, the control task comprises the control of three independent first-order systems which is solved by pure proportional controllers, respectively:

$$
\begin{aligned}
& \tilde{u}_{1}=k_{1} \cdot\left(x_{1 d}-x_{1}\right) \\
& \tilde{u}_{2}=k_{2} \cdot\left(x_{2 d}-x_{2}\right) \\
& \tilde{u}_{3}=k_{3} \cdot\left(x_{3 d}-x_{3}\right)
\end{aligned}
$$

Herein the controller parameters $k_{1}, k_{2}$ and $k_{3}$ could be chosen in a way that the outer loop is sufficiently fast but not too fast with respect to the inner loop attitude control. In a next step, these transformed input variables $\tilde{u}_{1}, \tilde{u}_{2}, \tilde{u}_{3}$ must be used to obtain the real input variables $x_{4 d}, x_{5 d}, x_{6 d}$ and $u_{1}$ by using (13). First it becomes obvious that any desired velocity vector can be achieved without any yaw rotation and therefore we can set $x_{6 d}=\psi_{d}=0$. Under this assumption it is shown in [6] that (13) can be solved analytically by calculating the inverse function of $f$ :

$$
\left(\begin{array}{c}
x_{4 d} \\
x_{5 d} \\
u_{1}
\end{array}\right)=\boldsymbol{f}^{-1}\left(\begin{array}{c}
\tilde{u}_{1} \\
\tilde{u}_{2} \\
\tilde{u}_{3}
\end{array}\right)
$$

\section{Overall Vehicle Control System}

The overall control system consist of the derived inner attitude and the outer velocity control loop. The command to the vehicle control system is a desired velocity vector given by $v_{x d}=x_{1 d}, v_{y d}=x_{2 d}, v_{z d}=x_{3 d}$. Then, (14) is used to calculate the respective values of the variables $\tilde{u}_{1}, \tilde{u}_{2}, \tilde{u}_{3}$ which are transferred by static inversion (15) into the values of the desired angles $x_{4 d}$ and $x_{5 d}$ as well as the input variable $u_{1}$. As discussed, the third desired angle is set to $x_{6 d}=0$. The desired angles are used to calculate $u_{2}^{*}, u_{3}^{*}, u_{4}^{*}$ and evaluation of (8) with the measured values of the angular rates $x_{7}, x_{8}, x_{9}$ and the nonlinear feedback yields the input variables $u_{2}, u_{3}, u_{4}$. Finally, (5) allows the calculation of the required angular rates of the rotors, namely $\omega_{1}, \omega_{2}, \omega_{3}$ and $\omega_{4}$. The main advantage of the overall control system is the fact that the feedback linearization and the controllers are comparatively easy to be implemented, while taking the full nonlinear behavior of the vehicle into account. That leads to a fast computation even on standard embedded microcontroller systems. Further details and simulation results are also given in [6], while experimental results will be presented here. The next step of designing a tracking and landing controller however requires a dynamic model of the controlled quadrotor, i.e. a dynamic model of the two nested control loops of attitude and velocity control loop. If we assume that the inner attitude control loops are sufficiently fast and could be approximated by a static system as discussed before, the overall vehicle control system consists of three independent velocity control loops which can be approximated by linear first-order system, respectively,

$$
\frac{V_{x}(s)}{V_{x d}(s)}=\frac{X_{1}(s)}{X_{1 d}(s)} \approx \frac{1}{T_{1} \cdot s+1}
$$

with $T_{i}=1 / k_{i}, i=1,2,3$, see (14). A simulation of the step response also supports this approximation, see Fig. 2 for the 
example of the step response with regard to the velocity $v_{x}$ in $\mathrm{x}$-direction. Similar results are obtained for the step response of the other two velocities. These first-order approximations of the controlled quadrotor UAV is now used for the design of the tracking and landing controllers.

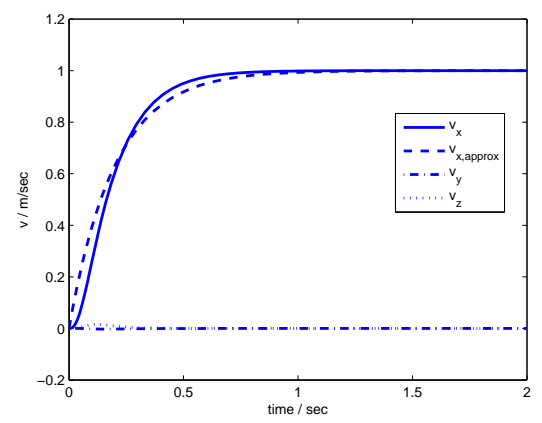

Fig. 2. Step response with regard to $v_{x}$ of the controlled quadrotor.

\section{Automatic Landing on a Mobile Platform}

In the following we consider the problem that a quadrotor UAV stabilized via the previously described vehicle control system should land on a moving platform. The platform is moving on the surface of the underlying terrain at an altitude of $z_{s}(t)$ with regard to the inertial frame. The overall tracking and landing procedure can be decomposed into two independent control tasks: a tracking procedure in a pure $\mathrm{x}-\mathrm{y}-$ plane and an altitude control problem in pure z-direction. In the pure $x-y$-plane, only the planar mappings of the center of mass of the quadrotor and the platform and their respective motions are considered. The 2D-tracking controller has the task to reduce the planar distance between the quadrotor and the platform in this two-dimensional plane to zero and to maintain the zero distance even if disturbances occur. In parallel, an altitude controller has the task to achieve and stabilize required setpoints of the quadrotor's altitude over ground. During a first approaching phase where the planar distance between the quadrotor and the platform is above a threshold, the setpoint of the altitude over ground is set to a safety value, e.g. $5 \mathrm{~m}$. If the planar distance decreases below the defined threshold, e.g. $0.5 \mathrm{~m}$, the final landing procedure starts and the required setpoint of the altitude over ground is set to zero.

The result of the altitude controller is a desired velocity component in z-direction, i.e. $\dot{z}_{d}=x_{3 d}$ for the underlying vehicle controller while the result of the 2D-tracking controller are the two components of the desired velocity vector in $\mathrm{x}$ and y-direction, i.e. $\dot{x}_{d}=x_{1 d}, \dot{y}_{d}=x_{2 d}$. Finally, the results of these two controllers form the overall desired velocity vector which is commanded to the vehicle controller. In the following, first the altitude controller is derived, then we discuss the developed 2D-tracking controller.

\section{A. Altitude Control}

The general task of the altitude control system is to achieve and maintain a desired altitude reference which can be either the constant altitude over ground during the approaching phase or a zero altitude over ground during the final landing phase. If $z$ is the altitude of the quadrotor UAV and $z_{s}$ is the current altitude of the surface (i.e. the platform) in the inertial frame, the difference $\Delta z=z-z_{s}$ is the relative altitude of the UAV over ground. The current desired altitude over ground commanded by the overall landing control is the value $\Delta z_{d}$. Now we assume that the dynamic behavior of the controlled quadrotor UAV in $z$-direction can be approximated by a first-order system as discussed in section 3 , i.e.

$$
F_{Q}(s)=\frac{V_{z}(s)}{V_{z d}(s)}=\frac{X_{3}(s)}{X_{3 d}(s)} \approx \frac{1}{T_{3} \cdot s+1}
$$

If a linear altitude controller with transfer function $F_{R, z}(s)$ is chosen, the structure of the resulting closed altitude control loop can be depicted as shown in Fig. 3. Herein the altitude of

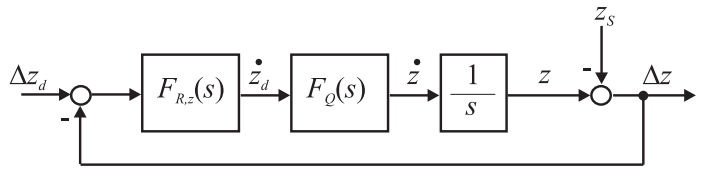

Fig. 3. Altitude control loop.

the surface $z_{s}$ is considered as a non-measurable disturbance, however the quadrotor is able to measure the current altitude over ground $\Delta z$ with a suitable sensor system. In the literature some solutions based on ultrasonic, optical or laser sensors have already been proposed for this measurement problem, see e.g. [7] and [8]. It becomes obvious from the structure of the altitude control loop shown in Fig. 3 that a PD-controller can be applied in order to solve the control task:

$$
F_{R, z}(s)=K \cdot\left(1+T_{C} \cdot s\right)
$$

The parameters of the controller are adjusted in a way that closed loop has zero overshoot (in order to avoid collisions with the platform during landing) and is sufficiently fast. The reference altitude $\Delta z_{d}$ is set to the desired safety altitude in the approach phase and set to zero in the landing phase.

\section{B. Nonlinear 2D-Tracking Controller}

The main goal of the guidance controller is to minimize the distance to a moving platform and to track this platform in the pure $x-y$-plane. For that purpose we consider a platform that is moving with the two velocity components $v_{P x}$ and $v_{P y}$ in $\mathrm{x}$ - and $\mathrm{y}$-direction, respectively. The quadrotor is moving with the two velocity components $v_{Q x}$ and $v_{Q y}$, where the dynamics between the desired velocities $v_{Q x d}, v_{Q y d}$ and the actual velocities is given by a firstorder system according to (16). The engagement geometry is depicted in Fig. 4, where $\sigma$ is the line-of-sight angle and $R$ is the distance or range between the quadrotor and the moving platform. It can be derived from classical missile guidance problems, see e.g. also [9], that the relative kinematics can 


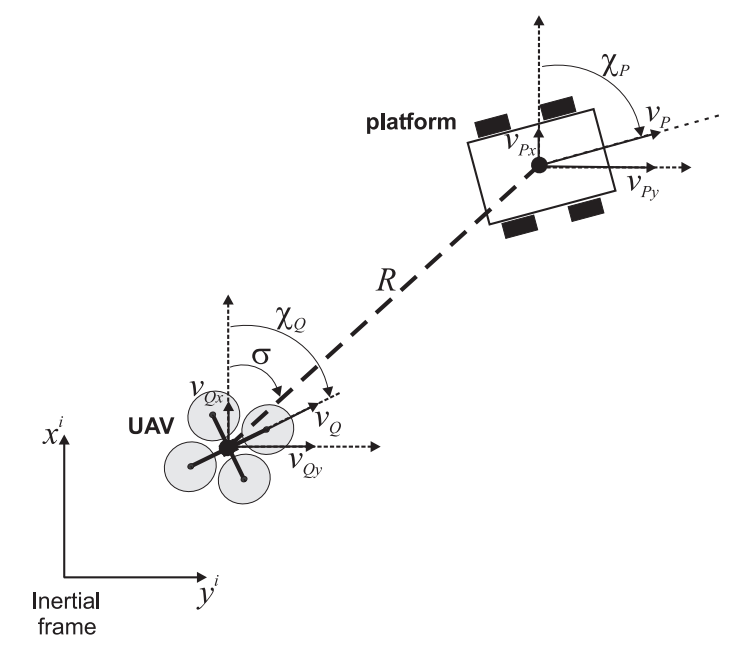

Fig. 4. Engagement geometry of quadrotor and mobile platform.

be described by the two differential equations:

$$
\begin{aligned}
\dot{R} & =v_{P x} \cos \sigma+v_{P y} \sin \sigma-v_{Q x} \cos \sigma-v_{Q y} \sin \sigma \\
\dot{\sigma} & =\frac{1}{R}\left(v_{P y} \cos \sigma-v_{P x} \sin \sigma-v_{Q y} \cos \sigma+v_{Q x} \sin \sigma\right)
\end{aligned}
$$

If we now again define a state variable model with the four state variables $x_{1}=R, x_{2}=\sigma, x_{3}=v_{Q x}, x_{4}=v_{Q y}$, the input variables $u_{1}=v_{Q x d}, u_{2}=v_{Q y d}$ and the two measurable disturbance variables $d_{1}=v_{P x}, d_{2}=v_{P y}$, we finally obtain from (16), (17):

$$
\begin{aligned}
& \dot{x}_{1}=-x_{3} \cos x_{2}-x_{4} \sin x_{2}+d_{1} \cos x_{2}+d_{2} \sin x_{2} \\
& \dot{x}_{2}=\frac{1}{x_{1}}\left(x_{3} \sin x_{2}-x_{4} \cos x_{2}-d_{1} \sin x_{2}+d_{2} \cos x_{2}\right) \\
& \dot{x}_{3}=-\left(1 / T_{1}\right) \cdot x_{3}+\left(1 / T_{1}\right) \cdot u_{1} \\
& \dot{x}_{4}=-\left(1 / T_{2}\right) \cdot x_{4}+\left(1 / T_{2}\right) \cdot u_{2}
\end{aligned}
$$

For the design of a suitable controller we first consider a suitable operating point. This is the state where the range and line-of-sight angle are zero and the quadrotor moves in accordance with the platform, i.e. $x_{1}=0, x_{2}=0, x_{3}=d_{1}, x_{4}=$ $d_{2}$. We define the Lyapunov function $V\left(x_{1}, x_{2}, x_{3}, x_{4}\right)$ which is $C^{1}$ and positive definite around the operating point:

$V\left(x_{1}, x_{2}, x_{3}, x_{4}\right)=0.5 \cdot\left(x_{1}^{2}+x_{2}^{2}+\left(x_{3}-d_{1}\right)^{2}+\left(x_{4}-d_{2}\right)^{2}\right)$

Now we calculate the first derivative of $V$ using (18) and assuming that the platform moves with a constant velocity:

$$
\begin{aligned}
\dot{V} & =x_{1} \dot{x}_{1}+x_{2} \dot{x}_{2}+\left(x_{3}-d_{1}\right) \dot{x}_{3}+\left(x_{4}-d_{2}\right) \dot{x}_{4} \\
& =-\frac{x_{3}^{2}}{T_{1}}-\frac{x_{4}^{2}}{T_{2}}+x_{3} f_{1}+x_{4} f_{2}+d_{1} f_{3}+d_{2} f_{4}
\end{aligned}
$$

with

$$
\begin{aligned}
f_{1} & =-x_{1} \cos x_{2}+\frac{x_{2}}{x_{1}} \sin x_{2}+\frac{1}{T_{1}} u_{1}+\frac{1}{T_{1}} d_{1} \\
f_{2} & =-x_{1} \sin x_{2}-\frac{x_{2}}{x_{1}} \cos x_{2}+\frac{1}{T_{2}} u_{2}+\frac{1}{T_{2}} d_{2} \\
f_{3} & =x_{1} \cos x_{2}-\frac{x_{2}}{x_{1}} \sin x_{2}-\frac{1}{T_{1}} u_{1} \\
f_{4} & =x_{1} \sin x_{2}+\frac{x_{2}}{x_{1}} \cos x_{2}-\frac{1}{T_{2}} u_{2}
\end{aligned}
$$

This derivative must be negative definite in order to guarantee that the operating point is asymptotically stable. Using (22) and (23), we first set $f_{3}=-\left(1 / T_{1}\right) \cdot d_{1}$ and $f_{4}=-\left(1 / T_{2}\right) \cdot d_{2}$ which yields

$$
\begin{aligned}
& u_{1}=d_{1}+T_{1} x_{1} \cos x_{2}-T_{1} \frac{x_{2}}{x_{1}} \sin x_{2} \\
& u_{2}=d_{2}+T_{2} x_{1} \sin x_{2}+T_{2} \frac{x_{2}}{x_{1}} \cos x_{2}
\end{aligned}
$$

Inserting this in $f_{1}, f_{2}$ using (23) leads to $f_{1}=\left(2 / T_{1}\right) \cdot d_{1}$ and $f_{2}=\left(2 / T_{2}\right) \cdot d_{2}$ which finally results in

$$
\dot{V}=-\frac{1}{T_{1}}\left(x_{3}-d_{1}\right)^{2}-\frac{1}{T_{2}}\left(x_{4}-d_{2}\right)^{2}
$$

This proofs that the derivative of $V$ is negative definite and the operating point is asymptotically stable if the tracking control law (24), (25) is applied. However, in order to obtain a limited control input, we set $u_{1}=d_{1}, u_{2}=d_{2}$ if the range $x_{1}$ becomes smaller than a defined very small threshold.

In order to apply this 2D-tracking controller, the range $x_{1}=R$, the line-of-sight angle $x_{2}=\sigma$ as well as the velocity components $d_{1}=v_{P x}, d_{2}=v_{P y}$ of the platform must be measured. Both $R$ and $\sigma$ can be easily calculated if the positions of the quadrotor and the platform in the inertial frame are measured. In addition it is assumed that the platform also measures its velocity components. Both position and velocity components of the platform are transmitted via communication to the quadrotor, resulting in a cooperative approach. Regarding the measurements, a DGPS is applied for the determination of the positions, respectively, during the approach phase. However, more accurate measurements are necessary during the landing phase. There are some possible solutions for this problem such as a vision based or ultrasonic based sensor system, see e.g. [7] and [8]. The velocity components of the platform could be measured with a suitable inertial measurement unit onboard. Since the main focus of this work is on the development of the control system, we do not go into further details of the measurements but describe some first simulation and experimental results in the next chapter.

\section{Simulation AND EXPERIMENTAL RESUltS}

In order to evaluate the derived vehicle and landing control system, an experimental prototype of the quadrotor has been designed and the dynamic model (6) of this quadrotor has been derived by identification of the system parameters like inertias, dimensions etc., see also [6] for a more detailed description. This dynamic model then has been implemented 
in MATLAB/SIMULINK for the simulative evaluation of the overall control system. The simulation results of the underlying vehicle control system are already shown in [6], therefore we first present some results of the vehicle controller obtained from experimental test flights with the quadrotor prototype. In the experiment the control goal was the stabilization of a hovering state, i.e. $\boldsymbol{v}_{d}=\mathbf{0}$ and $\boldsymbol{\Omega}_{d}=\mathbf{0}$, starting from any initial deviations and compensating for any external disturbances. The obtained control result is shown in Fig. 5 as a time plot of all angles of the quadrotor. After a very short transition phase the hovering state is reached and maintained. The small constant deviation of the yaw angle results from a slight misalignment of the inertial measurement unit. It becomes obvious from Fig. 5 that external disturbances at 35 seconds of the roll angle, at 45 seconds of the pitch angle and at 50 seconds at the yaw angle are completely compensated.
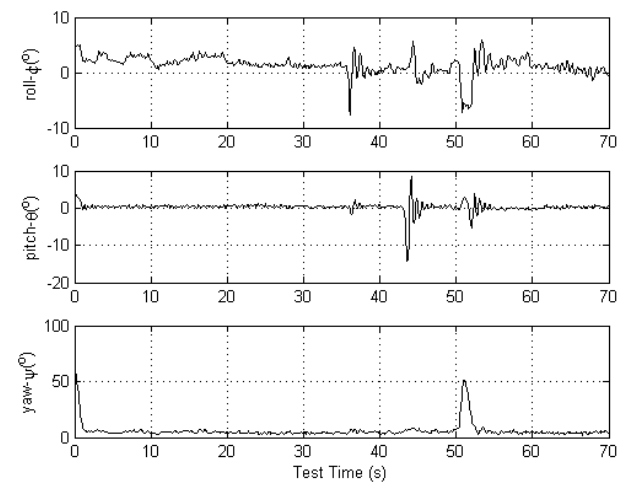

Fig. 5. Experimental results of the vehicle control system.

The overall landing control system is not yet implemented in the experimental quadrotor prototype and is therefore evaluated in simulations. In the simulation, the platform is initially assumed to be located at $\left(x_{P 0}=100 \mathrm{~m}, y_{P 0}=100\right.$ $\mathrm{m}$ and moving with a constant speed of $V_{P x}=-0.5 \mathrm{~m} / \mathrm{sec}$ in the $x-y$-plane. The quadrotor is initially located at $\left(x_{Q 0}=50\right.$ $\mathrm{m}, y_{Q 0}=50 \mathrm{~m}$ in the inertial frame at an altitude of $\Delta z_{0}=5$ $\mathrm{m}$. The obtained control result of the overall landing control system is depicted in Fig. 6. Diagram (a) shows a top view of the 2D-engagement in which the quadrotor starts from the initial position, tracks the path of the moving platform and finally lands on the platform. Diagram (b) shows the altitude of the quadrotor which descends from the initial altitude over ground until the final landing.

\section{Conclusion And Future Works}

This paper presents an overall control system for the automatic landing of a quadrotor UAV on a moving platform. Herein, the vehicle control system comprises a nonlinear inner loop attitude control and an outer loop velocity control system based on static inversion. The landing controller consists of a linear altitude controller and a nonlinear 2Dtracking controller. The dynamic model of the quadrotor and the proposed landing control system are implemented
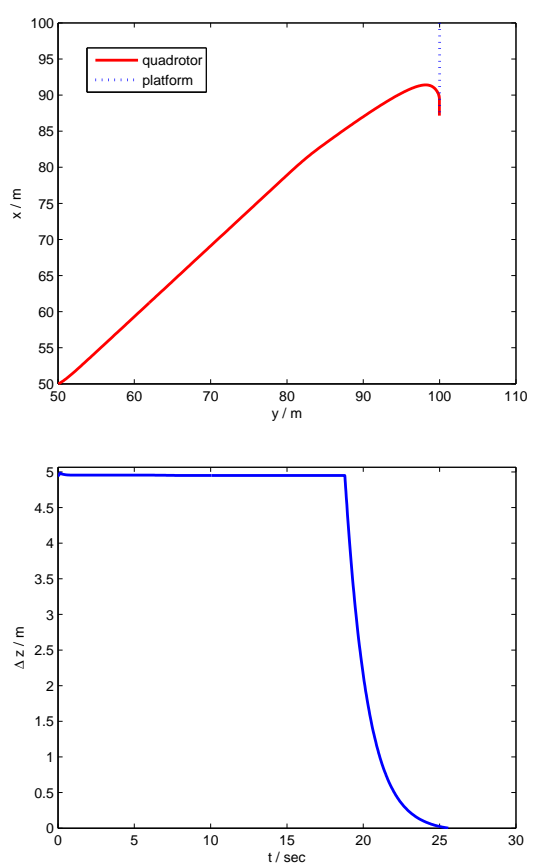

Fig. 6. Simulation of the overall landing control system.

in a MATLAB/SIMULINK simulation which proofs the efficiency of the overall control result. The vehicle control system is finally realized in an experimental prototype and first test flights underline the performance of this novel nonlinear approach. In our ongoing work we are currently also implementing the landing control system as well as the necessary sensors in the UAV prototype.

\section{REFERENCES}

[1] S. Bouabdallah, R. Siegwart. Backstepping and Sliding-mode Techniques Applied to an Indoor Micro Quadrotor. In Proc. of the IEEE International Conference on Robotics and Automation, 2005, pp. 2247 2252.

[2] A. Tayebi, S. McGilvray. Attitude Stabilization of a VTOL Quadrotor Aircraft. In IEEE Trans. on Control Systems Technology, 2006, Vol. 14, 2006, pp. $562-571$.

[3] P. Castillo, A. Dzul, R. Lozano. Real-time stabilization and tracking of a four-rotor mini rotorcraft. IEEE Trans. on Control Systems Technology, Vol.12, No. 4, July 2004, pp. 510 - 516.

[4] H. Voos. Nonlinear State-Dependent Riccati Equation Control of a Quadrotor UAV. In Proc. of the IEEE Conference on Control Applications, Munich, 2006.

[5] H. Voos. Nonlinear and Neural Network-based Control of a Small Four-Rotor Aerial Robot. In Proc. of the IEEE/ASME Int. Conference on Advanced Intelligent Mechatronics, Zurich, CH, 2007.

[6] H. Voos. Nonlinear Control of a Quadrotor Micro-UAV using Feedback-Linearization. In Proc. of the IEEE International Conference on Mechatronics (ICM 2009), Málaga, Spain, 14-17 April, 2009.

[7] S.L. Waslander et. al. Multi-agent quadrotor testbed control design: integral sliding mode vs. reinforcement learning. In Proc. of IEEE/RSJ International Conference on Intelligent Robots and Systems(IROS 2005), 2005, pp. 3712-3717.

[8] D.B. Barber, S.R. Griffiths, T.W. McLain and R.W. Beard. Autonomous Landing of Miniature Aerial Vehicles. Brigham Young University Press, Provo, UT.

[9] P. Zarchan. Tactical and Strategic Missile Guidance. AIAA Press, 2007. 